\title{
Prognostic and predictive value of the
}

\section{macroscopic growth pattern in patients}

undergoing curative resection of colorectal

\section{cancer: a single-institution retrospective cohort study of 4,080 Chinese patients}

This article was published in the following Dove Press journal:

Cancer Management and Research

\begin{abstract}
Xiao $\mathrm{Li}^{1,2}$
Qi Zhao',2

Bang $\mathrm{An}^{3}$

Jianni $\mathrm{Qi}^{2,4}$

Wenwen Wang ${ }^{1,2}$

Di Zhang ${ }^{1,2}$

Zhen $\mathrm{Li}^{1,2}$

Chengyong Qin ${ }^{1,2}$

'Department of Gastroenterology, Shandong Provincial Hospital Affiliated to Shandong University, Jinan 25002I, Shandong Province, People's Republic of China; ${ }^{2}$ Shandong Provincial Engineering and Technological Research Center for Liver Diseases Prevention and Control, Jinan 25002I, Shandong Province, People's Republic of China; ${ }^{3}$ Department of Cardiology, Central Hospital of Zibo, Zibo, People's Republic of China; ${ }^{4}$ Central Laboratory, Shandong Provincial Hospital Affiliated to Shandong University, Jinan 25002I, Shandong Province, People's Republic of China
\end{abstract}

Correspondence: Chengyong Qin Department of Gastroenterology, Shandong Provincial Hospital Affiliated to Shandong University, 324 Jingwu Road, Huaiyin District, Jinan 25002I, Shandong Province, People's Republic of China Tel +86 053। 68776912

Email chengyong_qin@।26.com
Purpose: The purpose of this study was to determine whether macroscopic growth patterns had an impact on the prognosis of colorectal cancer (CRC) patients with different tumor-nodemetastasis (TNM) stages and responses to chemotherapy in stage III patients.

Patients and methods: We retrospectively recruited 4,080 stage I-III CRC patients who underwent curative resection at Shandong Provincial Hospital affiliated to Shandong University. All patients were grouped by macroscopic growth patterns (expansive, infiltrative and ulcerative subtypes), and stage III patients were further divided into chemotherapy and nonchemotherapy groups. Kaplan-Meier methods, univariate and multivariate analyses and subset analyses were performed to assess the overall survival (OS), cancer-specific survival (CSS) and disease-free survival (DFS).

Results: Kaplan-Meier survival curves and univariate analyses revealed better OS (HR=0.731; 95\% CI $=0.584-0.916), \mathrm{CSS}(\mathrm{HR}=0.714 ; 95 \% \mathrm{CI}=0.548-0.932)$ and DFS (HR=0.722; 95\% $\mathrm{CI}=0.602-0.864)$ in the expansive subtype and worse $\mathrm{OS}(\mathrm{HR}=2.121 ; 95 \% \mathrm{CI}=1.457-3.088)$, CSS (HR=2.499; 95\% CI=1.664-3.753) and DFS (HR=2.360; 95\% CI=1.756-3.170) in the infiltrative subtype. Subset analyses based on the tumor-node-metastasis stage showed that the infiltrative subtype was associated with inferior DFS in stage II ( $\mathrm{HR}=2.357 ; 95 \% \mathrm{CI}=1.210-4.595)$ and stage III patients $(\mathrm{HR}=1.941 ; 95 \% \mathrm{CI}=1.394-2.702)$ and inferior OS and CSS in stage III patients $(\mathrm{HR}=1.805 ; 95 \% \mathrm{CI}=1.210-2.693$ and $\mathrm{HR}=1.981,95 \% \mathrm{CI}=1.280-3.065$, respectively $)$. In addition, multivariate Cox proportional hazard regression models revealed similar results. Furthermore, in stage III patients, the OS, CSS and DFS in both the expansive and ulcerative subtypes were significantly extended after the administration of chemotherapy (all, $P<0.001$ ). However, the OS, CSS and DFS in the infiltrative subtype did not change significantly after the administration of chemotherapy ( $P=0.486,0.290$ and 0.731 , respectively).

Conclusion: The macroscopic growth pattern was an independent prognostic factor among stage I-III CRC patients. The infiltrative subtype had the worst prognosis in stage III patients and did not display survival benefits from chemotherapy.

Keywords: colon cancer, infiltrative subtype, prognosis, TNM stage, chemotherapy

\section{Introduction}

Colorectal cancer (CRC) is the third most common cancer and a leading cause of cancer-related mortality worldwide. ${ }^{1,2}$ Over the past several decades, the outcomes 
of CRC patients have improved significantly owing to advanced surgical techniques and novel adjuvant chemotherapy regimens, but the 5-year survival rate remains $\sim 60 \%$. ${ }^{3,4}$ In clinical practice, a variety of approaches have been tried to systematically manage CRC to improve poor outcomes. Tumor-node-metastasis (TNM) staging is the most significant risk factor for $\mathrm{CRC},{ }^{5}$ and it is recommended that stage III and high-risk stage II patients undergo adjuvant chemotherapy. ${ }^{6}$ However, the prognosis and chemotherapeutic sensitivity of CRC in patients with the same TNM stage vary distinctly. TNM staging based on clinicopathological characteristics, such as tumor location ${ }^{7}$ and perineural invasion (PNI), ${ }^{8}$ has been widely investigated. However, to date, the optimal prognostic factor for $\mathrm{CRC}$ has not been discovered and further investigation is warranted. Therefore, additional sensitive predictive factors that can be easily acquired are needed to better evaluate the prognosis and response to chemotherapy of CRC and used as supplementary factors for TNM staging.

Macroscopic morphological features are common medical parameters that can easily be obtained from medical records and help classify CRC in China. Tumors are commonly classified into three morphological subtypes (expansive, infiltrative and ulcerative subtypes) according to gross observations based on the Chinese Standard for the Diagnosis and Treatment of Colorectal Cancer (2010), ${ }^{9}$ and the colonoscopic findings of these subtypes are shown in Figure 1.

Extensive studies have investigated the relationship between histological findings and clinical outcomes of $\mathrm{CRC}$, but no consensus has been reached on the prognostic and predictive value of macroscopic growth patterns in CRC. ${ }^{10}$ Previous studies have shown that the gross tumor configuration is a factor with no prognostic significance. ${ }^{11,12}$ However, accumulating evidence has demonstrated that CRC is characterized differently based on macroscopic morphological features, including biological behaviors, genetic features and prognosis. ${ }^{13,14}$ Yang et al found that in terms of prognosis, gross tumor type was correlated with disease-free interval. ${ }^{15}$ Chen et al divided CRC into two morphologies (massive and ulcerative/infiltrative morphologies) and found that the ulcerative/infiltrative morphology was an independent risk factor for apical node metastasis and thus was correlated with poor prognosis. ${ }^{16}$ Chian et al also divided CRC into two major groups (polypoid and ulcerative CRC) which displayed different genetic alterations and clinicopathological features. ${ }^{17}$ However, they excluded the infiltrative subtype or combined it with the ulcerative subtype. Few studies have compared the clinical survival and response to chemotherapy between the infiltrative subtype and other subtypes, which should not be neglected.

Thus, in the present study, expansive, infiltrative and ulcerative growth patterns were evaluated as distinct subtypes, and we compared the clinical, pathological and molecular features among them to investigate whether different macroscopic growth patterns were associated with the prognosis. Furthermore, for stage III CRC patients, we compared the prognosis of the chemotherapy group and the nonchemotherapy group with different macroscopic growth patterns to evaluate the predictive effect of macroscopic growth patterns for chemotherapeutic efficacy.

\section{Patients and methods}

\section{Patients}

A total of 4,080 qualified CRC patients who were treated at Shandong Provincial Hospital affiliated to Shandong University between March 2000 and July 2016 were retrospectively recruited. The inclusion criteria were as follows: 1) patients with pathologically confirmed carcinoma of the colon and rectum; 2) patients who underwent curative surgical resection; 3) patients who were 18-90 years old at the time of diagnosis and 4) patients with stage I-III CRC. The exclusion criteria were as follows: 1) patients with two or more asynchronously or synchronously primary tumors; 2) patients with synchronous distant metastases; 3 ) patients without data regarding macroscopic growth pattern or 4) patients who
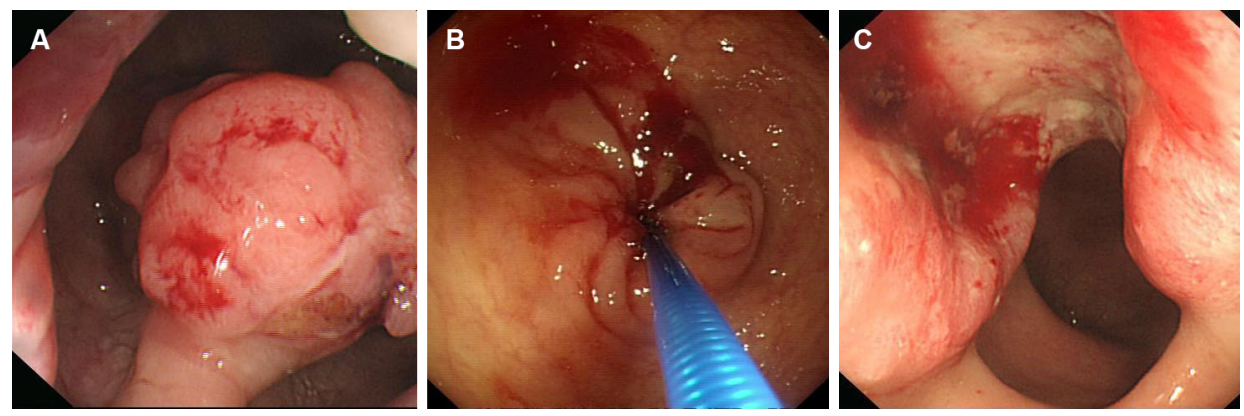

Figure I Colonoscopic findings of expansive, infiltrative and ulcerative growth patterns of colorectal cancer Notes: (A) expansive subtype, (B) infiltrative subtype and (C) ulcerative subtype. 
received neoadjuvant therapy. The seventh edition of the AJCC Cancer Staging Manual was used for tumor staging.

\section{Data collection}

The intact data, including clinicopathological features and demographic characteristics, were collected from the hospital database by trained staff. The data collection procedures were controlled for quality, and the data collected were standardized. The following parameters were recorded: gender, age at diagnosis, primary tumor location, tumor differentiation, TNM stage, histological subtype, macroscopic growth pattern, presence of venous invasion, presence of PNI, presence of tumor deposits and chemotherapeutic treatment.

\section{Ethical approval and informed consent}

The study protocol was approved by the Ethics Committee of Shandong Provincial Hospital affiliated to Shandong University. Written informed consent was obtained, and all the methods used were performed in accordance with the relevant guidelines and regulations from the committee.

\section{Follow-up}

Follow-up data were collected from the tumor registry database and the total death database of the Shandong Center for Disease Control and Prevention by the School of Public Health, Shandong University. The overall survival (OS), cancer-specific survival (CSS) and disease-free survival (DFS) were selected as primary endpoints. The OS was defined as the time from initial surgical treatment to death due to any cause. The CSS was calculated from the date of surgery to the date of CRC-specific death. Deaths attributed to other causes were censored. The DFS was defined as the period from surgical resection to first recurrence or metastasis or end of life. Patients who were still alive at the last follow-up were censored. The median duration of follow-up for all patients was 39.11 months (range 0.03-207.5 months).

\section{Statistical analysis}

Continuous variables are reported as the median and range, and categorical variables are presented as frequencies and percentages unless otherwise stated. The chi-square test $\left(\chi^{2}\right)$ test or the Fisher's exact test was utilized to compare differences in the distributions and proportions of the demographic and clinicopathological variables by macroscopic growth patterns. Survival curves of OS, CSS and DFS were generated by the Kaplan-Meier method and compared by the log-rank test. Multicollinearity diagnosis was performed before constructing the multivariate regression model. The association between macroscopic growth patterns and clinical outcomes (OS, CSS and DFS) was evaluated, and the corresponding HRs and 95\% CIs for prognostic factors were obtained from the univariate and multivariate Cox proportional hazard models. The effect of macroscopic growth patterns on OS, CSS and DFS was also assessed in subset analyses that were stratified by TNM stages and several other baseline variables. All statistical analyses were performed with SPSS software version 22.0 (SPSS Inc, Chicago, IL, USA). Statistical significance was set at a two-sided $P$-value $<0.05$.

\section{Results}

\section{Patient characteristics}

Patients' baseline demographic and clinicopathological parameters are summarized in Table 1. Of the 4,080 CRC patients recruited in this study, $60.27 \%$ were men and $39.73 \%$ were women. The patients were most commonly diagnosed between the ages of 51 and 75 years $(68.33 \%)$. Overall, $16.23 \%$ of the tumors were poorly differentiated, and $74.17 \%$ were moderately differentiated. The most common primary tumor location was rectal cancer (or RECC, $62.18 \%$ ), followed by left colon cancer (or LCC, splenic flexure to rectosigmoid, 19.34\%) and right colon cancer (or RCC, cecum to transverse, $18.43 \%$ ). The majority of patients were diagnosed with stage II CRC $(42.50 \%)$, followed by stage III CRC (41.86\%). Overall, $84.34 \%$ of the patients had nonmucinous tumors, $2.06 \%$ had venous invasion, $1.94 \%$ had PNI and 3.63\% exhibited tumor deposits.

Among all patients, the percentage of CRC patients with ulcerative, expansive and infiltrative macroscopic growth patterns was $77.38 \%(n=3157), 20 \%(n=816)$ and $2.62 \%(n=107)$, respectively. Compared with infiltrative and ulcerative subtypes, the expansive subtype was associated with higher frequencies of favorable features, such as welldifferentiated tumors ( $4.04 \%$ vs. $1.87 \%$ and $1.65 \%)$ and stage I tumors (38.36\% vs. $4.67 \%$ and $10.14 \%$, respectively), and lower frequencies of poor differentiation ( $12.38 \%$ vs. $21.50 \%$ and $17.04 \%$, respectively), lymph node invasion $(28.68 \%$ vs. $64.49 \%$ and $44.50 \%$ ), venous invasion $(1.72 \%$ vs. $7.48 \%$ and $1.96 \%)$, PNI ( $0.49 \%$ vs. $2.80 \%$ and $2.28 \%$, respectively), tumor deposits $(2.82 \%$ vs. $12.15 \%$ and $3.55 \%)$ and stage III tumors (28.68\% vs. $64.49 \%$ and $44.5 \%)$. However, the infiltrative subtype was associated with higher frequencies of the mucinous subtype (24.30\% vs. $19.98 \%$ and $14.25 \%$ ) than ulcerative and expansive subtypes. Patients' characteristics stratified by TNM stage and macroscopic growth pattern are listed in Table 2. 
Table I Demographic and clinicopathological features of patients with colorectal cancer

\begin{tabular}{|c|c|c|c|c|c|}
\hline Features & $\begin{array}{l}\text { All } \\
(N=4,080), n(\%)\end{array}$ & $\begin{array}{l}\text { Expansive } \\
(\mathrm{N}=8 \mid 6), \mathrm{n}(\%)\end{array}$ & $\begin{array}{l}\text { Infiltrative } \\
(\mathrm{N}=107), \mathrm{n}(\%)\end{array}$ & $\begin{array}{l}\text { Ulcerative } \\
(N=3,157), \text { n (\%) }\end{array}$ & $P$-value* \\
\hline \multicolumn{6}{|l|}{ Gender } \\
\hline Male & $2,459(60.27)$ & 44 I (54.04) & $62(57.94)$ & $1,956(61.96)$ & $<0.001$ \\
\hline Female & I,62I (39.73) & $375(45.96)$ & $45(42.06)$ & $\mathrm{I}, 20 \mathrm{I}(38.04)$ & \\
\hline \multicolumn{6}{|l|}{ Age, years } \\
\hline$<50$ & 907 (22.23) & 199 (24.39) & $33(30.84)$ & $675(21.38)$ & 0.175 \\
\hline $5 I-65$ & I,776 (43.53) & 339 (4I.54) & $40(37.38)$ & I,397 (44.25) & \\
\hline $66-75$ & I,0I2 (24.80) & $203(24.88)$ & $23(21.50)$ & $786(24.90)$ & \\
\hline$>75$ & $385(9.44)$ & $75(9.19)$ & II (I0.28) & $299(9.47)$ & \\
\hline \multicolumn{6}{|l|}{ Differentiation } \\
\hline Well & $87(2.13)$ & $33(4.04)$ & $2(1.87)$ & $52(1.65)$ & $<0.001$ \\
\hline Moderate & $3,026(74.17)$ & $592(72.55)$ & $64(59.81)$ & $2,370(75.07)$ & \\
\hline Poor & $662(16.23)$ & $101(12.38)$ & $23(21.50)$ & $538(17.04)$ & \\
\hline Unknown & $305(7.48)$ & $90(11.03)$ & $18(16.82)$ & $197(6.24)$ & \\
\hline \multicolumn{6}{|l|}{ Histological type } \\
\hline Nonmucinous & $3,44 \mid(84.34)$ & $653(80.02)$ & 81 (75.70) & 2,707 (85.75) & $<0.001$ \\
\hline Mucinous & $639(15.66)$ & $163(19.98)$ & $26(24.30)$ & $450(14.25)$ & \\
\hline \multicolumn{6}{|l|}{ Stage } \\
\hline 1 & $638(15.64)$ & $313(38.36)$ & $5(4.67)$ & $320(10.14)$ & $<0.001$ \\
\hline II & I,734 (42.50) & 269 (32.97) & $33(30.84)$ & I,432 (45.36) & \\
\hline III & I,708 (4I.86) & $234(28.68)$ & 69 (64.49) & I,405 (44.50) & \\
\hline \multicolumn{6}{|l|}{ Location } \\
\hline $\mathrm{RCC}$ & $752(18.43)$ & 144 (17.65) & $3 \mathrm{I}(28.97)$ & 577 (I8.28) & 0.022 \\
\hline LCC & 789 (19.34) & $157(19.24)$ & $25(23.36)$ & $607(19.23)$ & \\
\hline RECC & $2,537(62.18)$ & $514(62.99)$ & $5 I(47.66)$ & $1,972(62.46)$ & \\
\hline Unknown & $2(0.05)$ & $\mathrm{I}(0.12)$ & $0(0.00)$ & I $(0.03)$ & \\
\hline \multicolumn{6}{|l|}{ Venous invasion } \\
\hline Positive & $84(2.06)$ & $14(1.72)$ & $8(7.48)$ & $62(1.96)$ & $<0.001$ \\
\hline Negative & $3,996(97.94)$ & $802(98.28)$ & $99(92.52)$ & 3,095 (98.04) & \\
\hline \multicolumn{6}{|l|}{$\mathrm{PNI}$} \\
\hline Positive & $79(1.94)$ & $4(0.49)$ & $3(2.80)$ & $72(2.28)$ & 0.003 \\
\hline Negative & 4,001 (98.06) & $812(99.5 I)$ & $104(97.20)$ & 3,085 (97.72) & \\
\hline \multicolumn{6}{|l|}{ Tumor deposit } \\
\hline Present & 148 (3.63) & $23(2.82)$ & $13(12.15)$ & II 2 (3.55) & $<0.001$ \\
\hline Absent & $3,932(96.37)$ & $793(97.18)$ & $94(87.85)$ & $3,045(96.45)$ & \\
\hline
\end{tabular}

Notes: $* P$-values were calculated by the chi-square test. The $P$-value for significance was $<0.05$. Patients with unknown information were not included in the $\chi^{2}$-test. Abbreviations: RCC, right colon cancer; LCC, left colon cancer; RECC, rectal cancer; PNI, perineural invasion.

\section{Kaplan-Meier survival analyses by growth pattern and tumor stage}

For all TNM stages, Kaplan-Meier survival curves revealed significantly better OS, CSS and DFS in the expansive subtype and worse OS, CSS and DFS in the infiltrative subtype than in the ulcerative subtype (all, $P<0.001$; Figure $2 \mathrm{~A}-\mathrm{C}$ ). In the stage I subanalysis, there were no significantly differences among the three subtypes $(P=0.697,0.768$ and 0.759 , respectively; Figure 2D-F). In the stage II subanalysis, the infiltrative subtype had the worst DFS ( $P=0.033$; Figure $2 \mathrm{I})$, but similar OS and CSS $(P=0.682$ and 0.382 , respectively; Figure $2 \mathrm{G}$ and $\mathrm{H})$. In the stage III subanalysis, the infiltrative subtype had the worst OS, CSS and DFS $(P=0.008, P=0.003$ and $P<0.001$, respectively; Figure $2 \mathrm{~J}-\mathrm{L}$ ).
The 5-year survival rates of the expansive, infiltrative and ulcerative subtypes were $72 \%, 56 \%$ and $67 \%$, respectively. For stage I patients, the 5-year survival rates of the expansive and ulcerative subtypes were $86 \%$ and $83 \%$, respectively. For stage II patients, the 5-year survival rates of the expansive, infiltrative and ulcerative subtypes were $71 \%, 89 \%$ and $77 \%$, respectively. For stage III patients, the 5-year survival rates of the expansive, infiltrative and ulcerative subtypes were $58 \%, 44 \%$ and $55 \%$, respectively.

\section{Univariate and multivariate Cox analyses by growth pattern and tumor stage}

For all stages, the univariate Cox proportional hazard model of OS revealed an increased risk of mortality for the infiltrative 


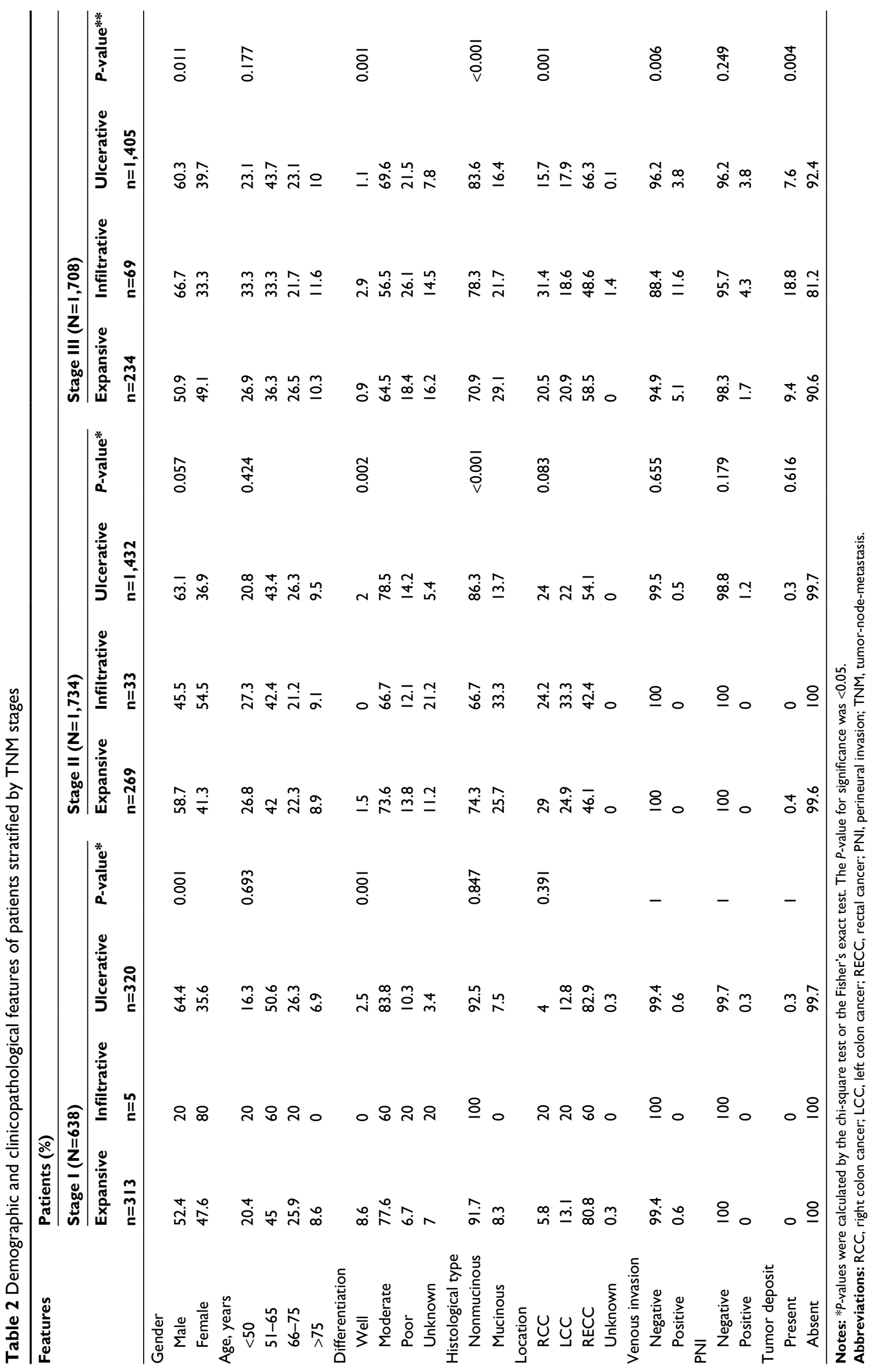




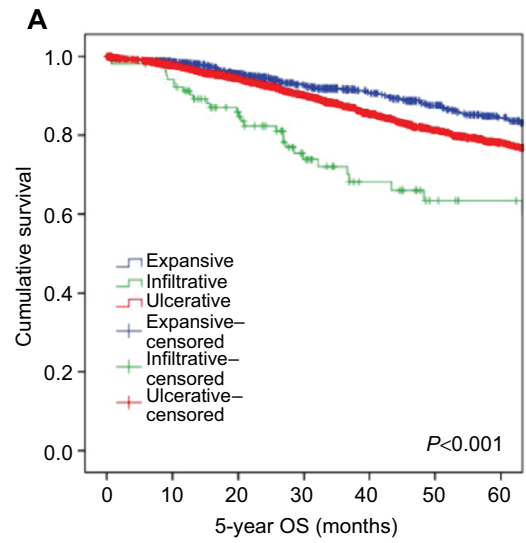

D

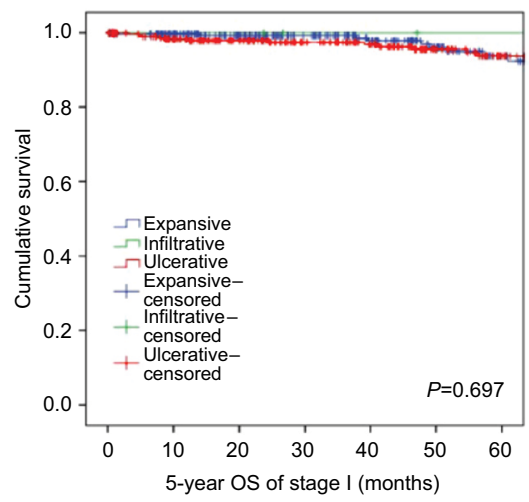

G

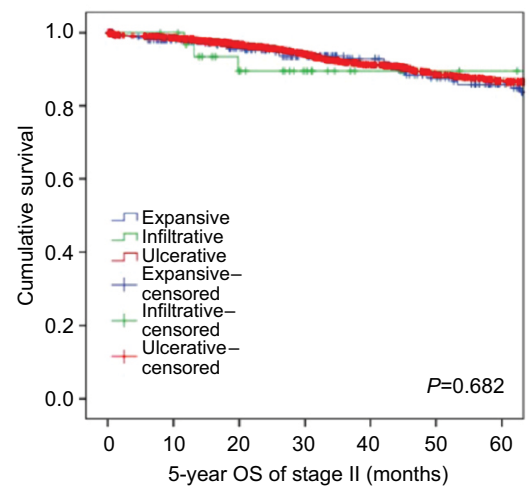

J

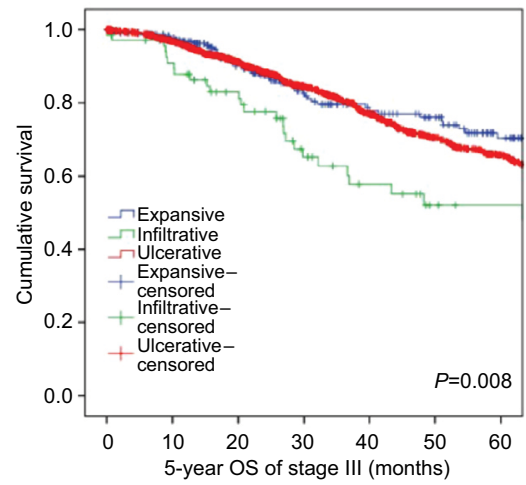

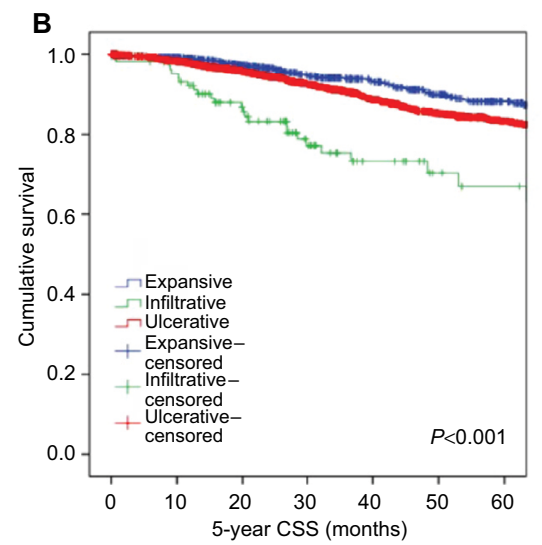

E

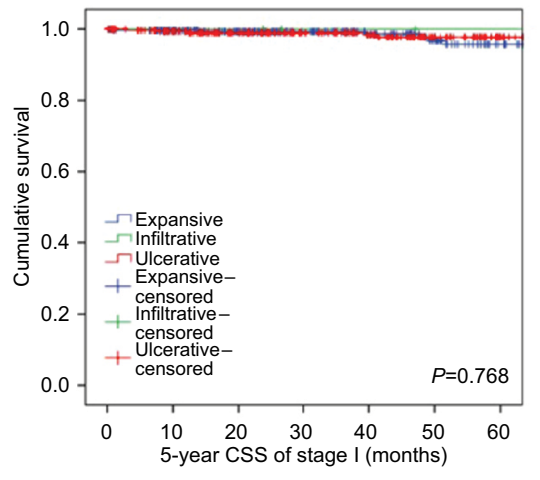

H

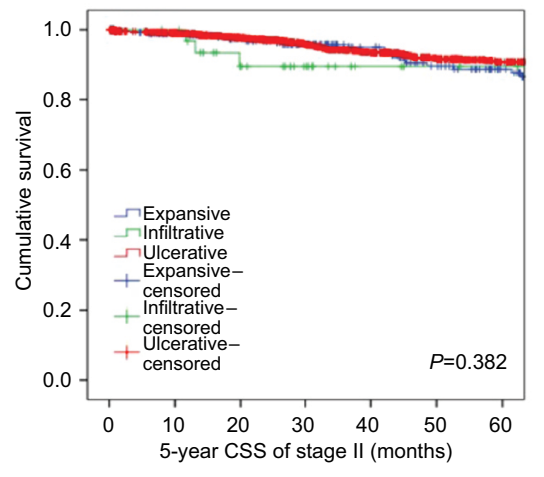

K

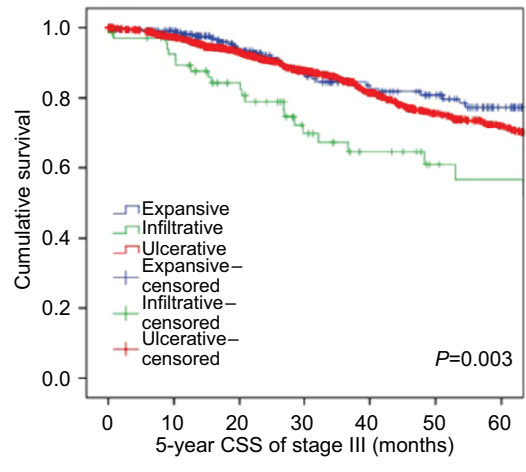

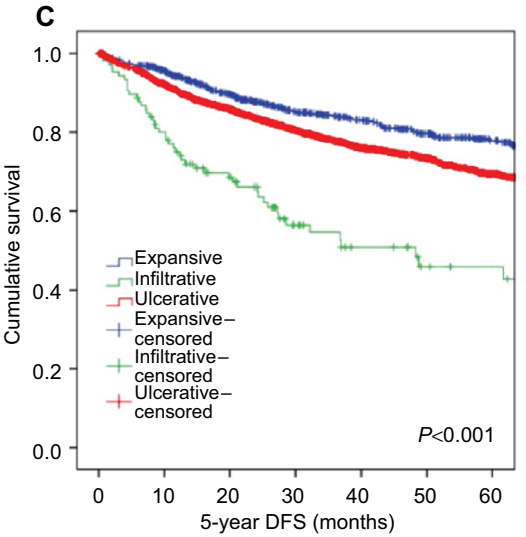

$$
\mathbf{F}
$$

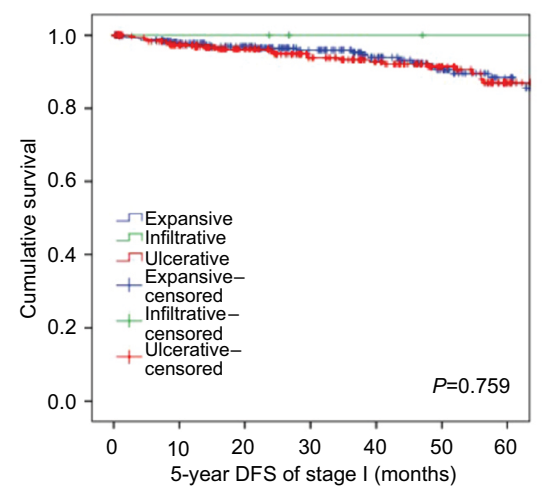

I

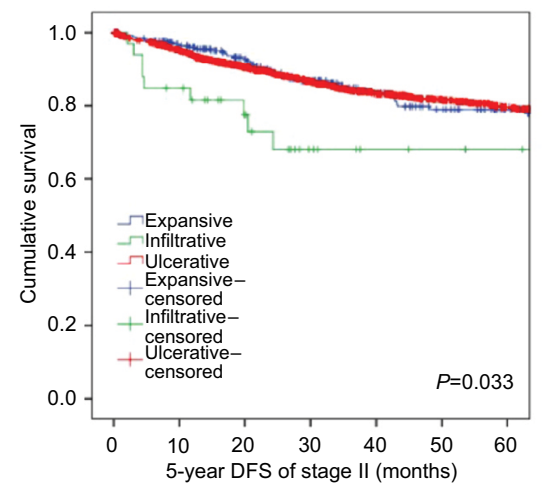

L

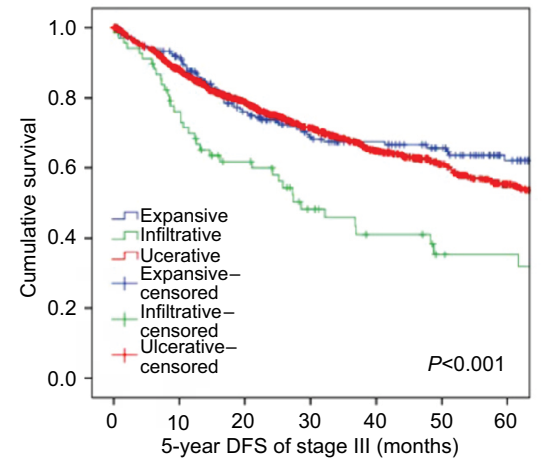

Figure 2 Kaplan-Meier survival analyses by the macroscopic growth pattern and the TNM stage.

Notes: (A) OS of all patients, (B) CSS of all patients, (C) DFS of all patients, (D) OS of stage I patients, (E) CSS of stage I patients, (F) DFS of stage I patients, (G) OS of stage II patients, (H) CSS of stage II patients, (I) DFS of stage II patients, (J) OS of stage III patients, (K) CSS of stage III patients and (L) DFS of stage III patients. Abbreviations: TNM, tumor-node-metastasis; OS, overall survival; CSS, cancer-specific survival; DFS, disease-free survival. 
subtype $(\mathrm{HR}=2.121 ; 95 \% \mathrm{CI}=1.457-3.088 ; P<0.001)$, and a decreased risk of mortality for the expansive subtype (HR $=0.731 ; 95 \% \mathrm{CI}=0.584-0.916 ; P=0.006$ ), compared with the ulcerative subtype. For stage III patients, the infiltrative subtype was also associated with a higher risk of mortality than the ulcerative subtype $(\mathrm{HR}=1.805 ; 95 \% \mathrm{CI}=1.210-2.693$; $P=0.004)$; however, no significant morphological specific differences affecting OS were observed in patients with stage I or II CRC. In addition, multivariate Cox proportional hazard regression models reached similar results after controlling for age; tumor differentiation; histological subtype and the presence of PNI, venous invasion and tumor deposits (Table 3).

Univariate Cox proportional hazard models of CSS revealed an increased risk of cancer-specific mortality for the infiltrative subtype $(\mathrm{HR}=2.499 ; 95 \% \mathrm{CI}=1.664-3.753$; $P<0.001)$, and a decreased risk of cancer-specific mortality for the expansive subtype compared with the ulcerative subtype for all stages $(\mathrm{HR}=0.714 ; 95 \% \mathrm{CI}=0.548-0.932$; $P=0.013$ ). For stage III patients, the infiltrative subtype was also associated with a higher risk of cancer-specific mortality than the ulcerative subtype $(\mathrm{HR}=1.981 ; 95 \% \mathrm{CI}=1.280-3.065$;
$P=0.002$ ), although no significant differences were observed for stage I and II patients. In addition, multivariate Cox proportional hazard regression models that were controlled for age; tumor differentiation; histological subtype and the presence of PNI, venous invasion and tumor deposits were constructed, and similar results were observed (Table 4).

Univariate Cox proportional hazard models of DFS revealed an increased risk of recurrence or metastasis for the infiltrative subtype $(\mathrm{HR}=2.360 ; 95 \% \mathrm{CI}=1.756-3.170$; $P<0.001$ ), and a decreased risk of recurrence or metastasis for the expansive subtype $(\mathrm{HR}=0.722 ; 95 \% \mathrm{CI}=0.602-0.864$; $P<0.001)$ compared with the ulcerative subtype for all stages. For stage II and III patients, the infiltrative subtype was also associated with a higher risk of recurrence or metastasis than the ulcerative subtype $(\mathrm{HR}=2.357 ; 95 \% \mathrm{CI}=1.210-4.595$; $P=0.012 ; \mathrm{HR}=1.941 ; 95 \% \mathrm{CI}=1.394-2.702 ; P<0.001$, respectively). In addition, multivariate Cox proportional hazard regression models reached similar results after controlling for gender; tumor differentiation; histological subtype and the presence of PNI, venous invasion and tumor deposits (Table 5).

Table 3 Univariate and multivariate analyses of OS in CRC patients with different TNM stages

\begin{tabular}{|c|c|c|c|c|c|c|c|c|c|c|c|c|}
\hline \multirow[t]{2}{*}{ Analysis type } & \multicolumn{3}{|c|}{ All stages $(\mathrm{N}=\mathbf{4 , 0 8 0})$} & \multicolumn{3}{|c|}{ Stage I $(n=638)$} & \multicolumn{3}{|c|}{ Stage II $(n=I, 734)$} & \multicolumn{3}{|c|}{ Stage III $(n=I, 708)$} \\
\hline & HR & $95 \% \mathrm{Cl}$ & $P$-value & HR & $95 \% \mathrm{Cl}$ & $P$-value & HR & $95 \% \mathrm{Cl}$ & $P$-value & HR & $95 \% \mathrm{Cl}$ & $P$-value \\
\hline \multicolumn{13}{|l|}{ Univariate } \\
\hline Expansive & 0.731 & $0.584-0.916$ & 0.006 & 0.776 & $0.363-1.662$ & 0.515 & 1.166 & $0.792-1.718$ & 0.436 & 0.904 & $0.667-1.226$ & 0.516 \\
\hline Infiltrative & 2.121 & $1.457-3.088$ & $<0.001$ & I & 1 & I & 1.297 & $0.412-4.076$ & 0.657 & 1.805 & $1.210-2.693$ & 0.004 \\
\hline Ulcerative & I & & & 1 & & & 1 & & & 1 & & \\
\hline \multicolumn{13}{|l|}{ Multivariate } \\
\hline Expansive & 0.754 & $0.60 \mathrm{I}-0.946$ & 0.015 & 0.878 & $0.402-1.917$ & 0.744 & 1.329 & $0.898-1.969$ & 0.155 & 0.878 & $0.646-1.194$ & 0.407 \\
\hline Infiltrative & 1.691 & I.I46-2.496 & 0.008 & I & 1 & 1 & 1.519 & $0.477-4.834$ & 0.479 & 1.527 & $|.009-2.3| 1$ & 0.045 \\
\hline Ulcerative & $\mathrm{I}$ & & & I & & & I & & & I & & \\
\hline
\end{tabular}

Note: a Cox regression model controlling for age, histological subtype, tumor differentiation, the presence of perineural invasion, venous invasion and tumor deposits.

Abbreviations: OS, overall survival; CRC, colorectal cancer; TNM, tumor-node-metastasis.

Table 4 Univariate and multivariate analyses of CSS in CRC patients with different TNM stages

\begin{tabular}{|c|c|c|c|c|c|c|c|c|c|c|c|c|}
\hline \multirow{2}{*}{$\begin{array}{l}\text { Analysis } \\
\text { type }\end{array}$} & \multicolumn{3}{|c|}{ All stages $(\mathrm{N}=4,080)$} & \multicolumn{3}{|c|}{ Stage I $(n=638)$} & \multicolumn{3}{|c|}{ Stage II $(n=I, 734)$} & \multicolumn{3}{|c|}{ Stage III $(n=I, 708)$} \\
\hline & HR & $95 \% \mathrm{Cl}$ & $P$-value & HR & $95 \% \mathrm{Cl}$ & $P$-value & HR & $95 \% \mathrm{Cl}$ & $P$-value & HR & $95 \% \mathrm{Cl}$ & $P$-value \\
\hline \multicolumn{13}{|l|}{ Univariate } \\
\hline Expansive & 0.714 & $0.548-0.932$ & 0.013 & 1.378 & $0.497-3.825$ & 0.538 & 1.242 & $0.785-1.964$ & 0.355 & 0.830 & $0.580-1.189$ & 0.310 \\
\hline Infiltrative & 2.499 & $1.664-3.753$ & $<0.001$ & 1 & 1 & 1 & 1.892 & $0.598-5.985$ & 0.278 & 1.981 & $1.280-3.065$ & 0.002 \\
\hline Ulcerative & 1 & & & 1 & & & 1 & & & 1 & & \\
\hline \multicolumn{13}{|l|}{ Multivariate $^{\mathrm{a}}$} \\
\hline Expansive & 0.737 & $0.564-0.964$ & 0.026 & 1.594 & $0.564-4.506$ & 0.380 & 1.358 & $0.853-2.162$ & 0.197 & 0.812 & $0.565-1.167$ & 0.260 \\
\hline Infiltrative & 1.998 & $1.309-3.050$ & 0.011 & 1 & 1 & 1 & 2.150 & $0.670-6.902$ & 0.198 & 1.640 & $1.04 \mathrm{I}-2.583$ & 0.033 \\
\hline Ulcerative & 1 & & & I & & & 1 & & & 1 & & \\
\hline
\end{tabular}

Note: ${ }^{2}$ Cox regression model controlling for age, tumor differentiation, histological subtype, the presence of perineural invasion, venous invasion and tumor deposits. Abbreviations: CSS, cancer-specific survival; CRC, colorectal cancer; TNM, tumor-node-metastasis. 
Table 5 Univariate and multivariate analyses of DFS in CRC patients with different TNM stages

\begin{tabular}{|c|c|c|c|c|c|c|c|c|c|c|c|c|}
\hline \multirow{2}{*}{$\begin{array}{l}\text { Analysis } \\
\text { type }\end{array}$} & \multicolumn{3}{|c|}{ All stages $(\mathrm{N}=\mathbf{4 , 0 8 0})$} & \multicolumn{3}{|c|}{ Stage I $(n=638)$} & \multicolumn{3}{|c|}{ Stage II $(n=I, 734)$} & \multicolumn{3}{|c|}{ Stage III $(n=I, 708)$} \\
\hline & HR & $95 \% \mathrm{Cl}$ & $P$-value & HR & $95 \% \mathrm{Cl}$ & $P$-value & $\overline{H R}$ & $95 \% \mathrm{Cl}$ & $P$-value & $\overline{H R}$ & $95 \% \mathrm{Cl}$ & $P$-value \\
\hline \multicolumn{13}{|l|}{ Univariate } \\
\hline Expansive & 0.722 & $0.602-0.864$ & $<0.001$ & 0.956 & $0.555-1.646$ & 0.870 & 0.090 & $0.724-1.356$ & 0.952 & 0.905 & $0.704-1.164$ & 0.438 \\
\hline Infiltrative & 2.360 & $1.756-3.170$ & $<0.001$ & 1 & 1 & I & 2.357 & $1.210-4.595$ & 0.012 & 1.941 & $1.394-2.702$ & $<0.001$ \\
\hline Ulcerative & 1 & & & & & & I & & & 1 & & \\
\hline \multicolumn{13}{|l|}{ Multivariate $^{\mathrm{a}}$} \\
\hline Expansive & 0.751 & $0.626-0.902$ & 0.002 & 1.075 & $0.6|8-1.87|$ & 0.798 & 1.039 & $0.757-1.426$ & 0.811 & 0.903 & $0.700-1.164$ & 0.430 \\
\hline Infiltrative & 1.886 & $1.388-2.562$ & $<0.001$ & 1 & 1 & I & 2.662 & $1.350-5.247$ & 0.005 & 1.607 & $1.140-2.266$ & 0.007 \\
\hline Ulcerative & I & & & I & & & I & & & 1 & & \\
\hline
\end{tabular}

Note: a Cox regression model controlling for gender, tumor differentiation, histological subtype, the presence of perineural invasion, venous invasion and tumor deposits. Abbreviations: DFS, disease-free survival; CRC, colorectal cancer; TNM, tumor-node-metastasis.

\section{Macroscopic growth pattern as a predictive factor for the selection of treatment pattern in stage III patients}

We further divided stage III patients into a chemotherapy group ( $77.34 \%)$ and a nonchemotherapy group $(\sim 22.66 \%)$ based on whether they received chemotherapy after complete resection. The Kaplan-Meier survival curves revealed that the OS, CSS and DFS of the expansive subtype (Figure 3A-C) and the ulcerative subtype (Figure 3G-I) could be extended significantly after the administration of chemotherapy (all, $P<0.001$ ), whereas the OS, CSS and DFS of the infiltrative subtype did not change significantly after the administration of chemotherapy $(P=0.486,0.290$ and 0.731 , respectively; Figure 3D-F).

\section{Subset analyses}

Multivariate Cox proportional hazard regression analyses were performed with various subsets. In the subset analyses of OS (Figure 4), CSS (Figure 5) and DFS (Figure 6), the infiltrative subtype was associated with worse prognosis than the ulcerative subtype for male patients, younger patients, patients with moderately differentiated cancer, patients with the nonmucinous subtype, patients with LCC or RECC, patients who were negative for venous invasion or PNI and patients without tumor deposits. By contrast, the expansive subtype was associated with better OS, CSS and DFS than the ulcerative subtype for patients with the nonmucinous subtype, patients with RECC, patients who were negative for venous invasion or PNI and patients without tumor deposits.

\section{Discussion}

The TNM staging system, which includes assessments of the local tumor extent, the status of regional lymph nodes and the presence of metastasis, is the gold standard in clinical practice. ${ }^{18}$ However, many patients with a lower TNM stage have a worse prognosis than those with a higher TNM stage, and not all patients benefit from chemotherapy. Thus, complementary predictive factors need to be identified to better evaluate the prognosis and response to chemotherapy of CRC.

In the past several decades, studies have focused on prognostic markers for CRC. However, controversy remains regarding the prognostic value of the macroscopic growth pattern. Most previous studies have excluded the infiltrative subtype or combined it with the ulcerative subtype. However, we thought there might be some differences in the mortality, recurrence or metastasis between the infiltrative subtype and other subtypes, which should not be neglected.

In the present study, all three macroscopic growth patterns were evaluated as distinct subtypes, and their demographic and clinicopathological features were compared. Our stagebased analysis revealed that compared with the infiltrative and ulcerative subtypes, the expansive subtype was associated with higher frequencies of favorable characteristics, including well-differentiation, lower TNM stage, less lymph node invasion, less venous invasion or PNI and absent tumor deposits. However, compared with the expansive and ulcerative subtypes, the infiltrative subtype consisted of a greater proportion of the mucinous subtype, indicating that the expansive subtype may have a better prognosis while the infiltrative subtype may have a worse prognosis. These findings may be attributed to the different biological behaviors of different morphological subtypes influencing tumorigenesis.

In addition, the Kaplan-Meier curves and univariate analyses of OS, CSS and DFS showed that the macroscopic growth pattern was a significant prognostic factor for CRC patients. Similar to previous studies, ${ }^{14}$ the ulcerative subtype is in an intermediate position between the best of the expansive subtype and the worst of the infiltrative subtype with regard to OS, CSS and DFS. However, in the subset analysis based on 

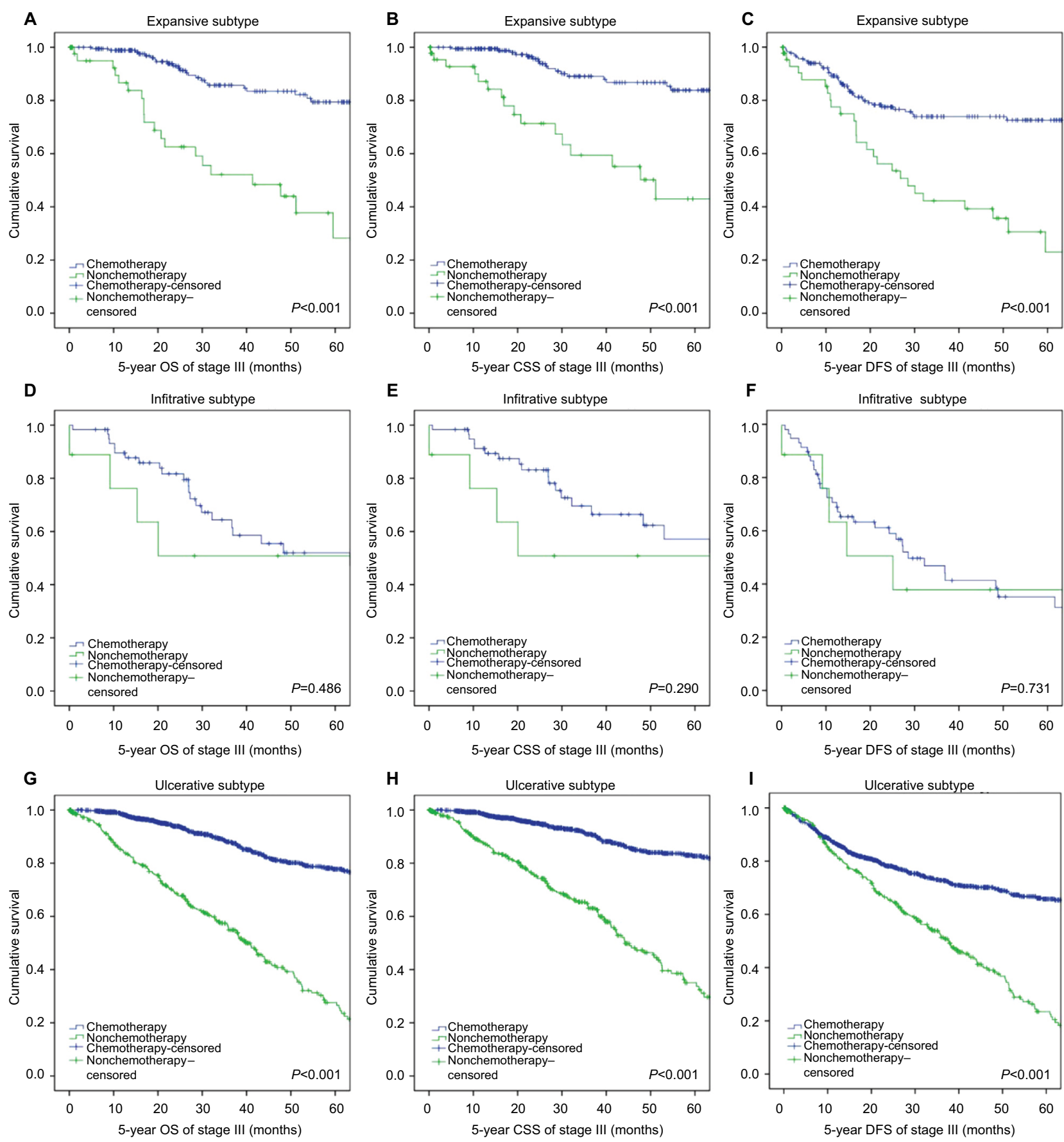

Figure 3 Kaplan-Meier survival analyses by chemotherapy and the macroscopic growth pattern in stage III patients.

Notes: (A) OS of the expansive subtype, (B) CSS of the expansive subtype, (C) DFS of the expansive subtype, (D) OS of the infiltrative subtype, (E) CSS of the infiltrative subtype, (F) DFS of the infiltrative subtype, (G) OS of the ulcerative subtype, (H) CSS of the ulcerative subtype and (I) DFS of the ulcerative subtype. Abbreviations: OS, overall survival; CSS, cancer-specific survival; DFS, disease-free survival.

the TNM stage, significant differences were observed between the infiltrative subtype and the ulcerative subtype for stage III patients in terms of OS, CSS and DFS and for stage II patients in terms of DFS. The fact that stage I CRC patients usually have good prognosis may account for the results.

Furthermore, after controlling for age; tumor differentiation; histological subtype and the presence of PNI, venous invasion and tumor deposits for OS and CSS, we observed significant differences in the OS and CSS between the three CRC subtypes. After controlling for gender; differentiation; histological subtype and the presence of venous invasion, PNI and tumor deposits for DFS, significant differences in the DFS were also observed. However, in the subset analyses based on the TNM stage, patients with the infiltrative subtype 


\section{Subgroup}

Male (expansive vs. ulcerative) Male (infiltrative vs. ulcerative) Female (expansive vs. ulcerative) Female (infiltrative vs. ulcerative) Age $<50$ years (expansive vs. ulcerative) Age $<50$ years (infiltrative vs. ulcerative) Age 51-65 years (expansive vs. ulcerative) Age 51-65 years (infiltrative vs. ulcerative) Age $66-75$ years (expansive vs. ulcerative) Age 66-75 years (infiltrative vs. ulcerative) Age $>75$ years (expansive vs. ulcerative) Age $>75$ years (infiltrative vs. ulcerative) Well differentiated (expansive vs. ulcerative) Well differentiated (infiltrative vs. ulcerative) Moderate differentiated (expansive vs. ulcerative) Moderate differentiated (infiltrative vs. ulcerative) Poor differentiated (expansive vs. ulcerative) Poor differentiated (infiltrative vs. ulcerative) Nonmucinous (expansive vs. ulcerative) Nonmucinous (infiltrative vs. ulcerative) Mucinous (expansive vs. ulcerative) Mucinous (infiltrative vs. ulcerative) RCC (expansive vs. ulcerative) RCC (infiltrative vs. ulcerative) LCC (expansive vs. ulcerative) LCC (infiltrative vs. ulcerative) RECC (expansive vs. ulcerative) RECC (infiltrative vs. ulcerative) Venous positive (expansive vs. ulcerative) Venous positive (infiltrative vs. ulcerative) Venous negative (expansive vs. ulcerative) Venous negative (infiltrative vs. ulcerative) Perineural positive (expansive vs. ulcerative) Perineural positive (infiltrative vs. ulcerative) Perineural negative (expansive vs. ulcerative) Perineural negative (infiltrative vs. ulcerative) Tumor deposit present (expansive vs. ulcerative) Tumor deposit present (infiltrative vs. ulcerative) Tumor deposit absent (expansive vs. ulcerative) Tumor deposit absent (infiltrative vs. ulcerative)

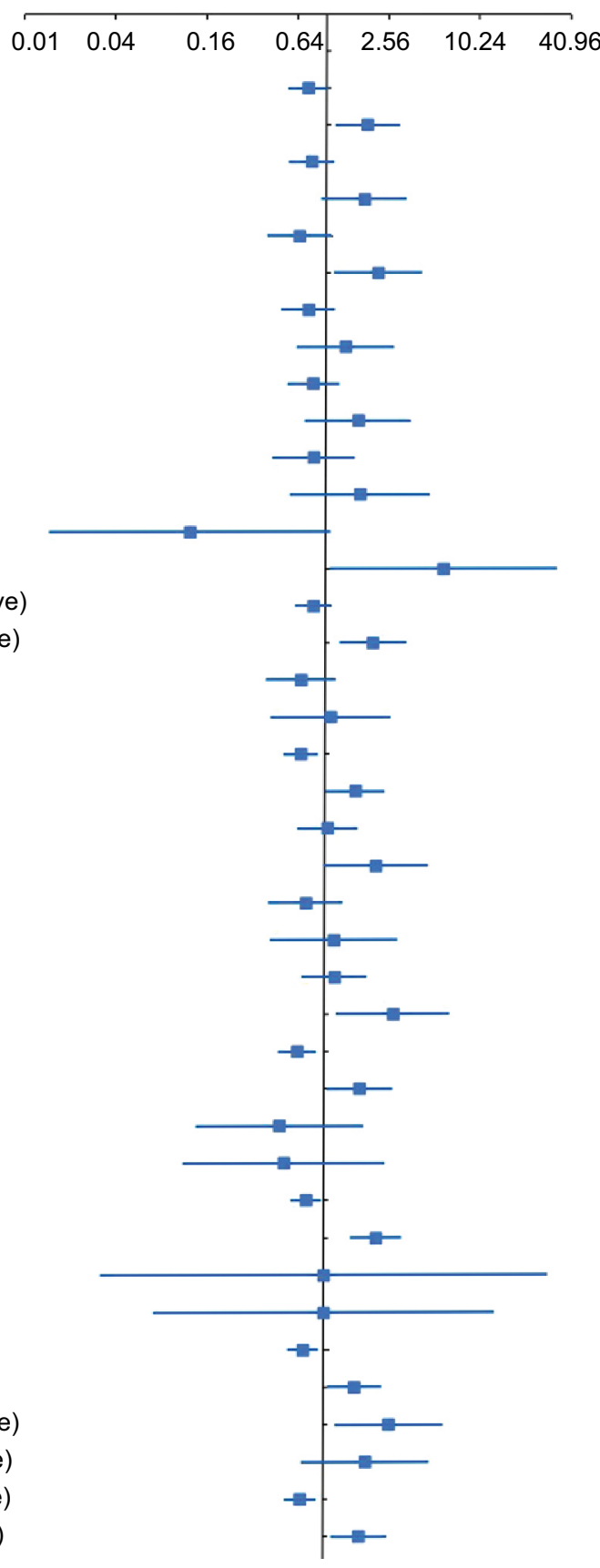
HRs $(95 \% \mathrm{Cl})$
$0.746(0.551-1.009)$
$1.858(1.136-3.04)$
$0.792(0.56-1.12)$
$1.765(0.92-3.385)$
$0.661(0.404-1.081)$
$2.189(1.115-4.296)$
$0.757(0.502-1.141)$
$1.333(0.636-2.793)$
$0.818(0.552-1.212)$
$1.621(0.727-3.615)$
$0.823(0.439-1.542)$
$1.673(0.576-4.857)$
$0.127(0.015-1.075)$
5.985 (1.063-33.695)
$0.828(0.63-1.088)$
$2.052(1.23-3.424)$
$0.69(0.406-1.173)$
$1.08(0.436-2.677)$
$0.687(0.527-0.896)$
$1.58(1.007-2.478)$
$1.039(0.659-1.64)$
$2.153(0.969-4.78)$
$0.746(0.424-1.314)$
$1.145(0.437-2.999)$
$1.154(0.708-1.881)$
$2.815(1.194-6.635)$
$0.661(0.493-0.886)$
$1.702(1.032-2.809)$
$0.508(0.142-1.818)$
$0.54(0.117-2.496)$
$0.763(0.605-0.963)$
$2.194(1.482-3.248)$
$1(0.034-29.493)$
$1(0.076-13.208)$
$0.727(0.578-0.916)$
$1.594(1.064-2.387)$
$2.684(1.188-6.063)$
$1.874(0.716-4.903)$
$0.699(0.551-0.888)$
$1.71(1.114-2.626)$

Figure 4 Adjusted HRs with $95 \% \mathrm{Cls}$ for OS comparing the infiltrative subtype and the expansive subtype to the ulcerative subtype in different subgroups. Abbreviations: OS, overall survival; RCC, right colon cancer; LCC, left colon cancer; RECC, rectal cancer.

had the worst DFS in stage II patients and the worst OS, CSS and DFS in stage III patients than other subtypes. In the subset analyses based on other clinicopathological factors, the infiltrative subtype was an independent prognostic factor in various subgroups, such as patients with the nonmucinous subtype, patients with RECC, patients who were negative for venous invasion or PNI and patients without tumor deposits, indicating that the macroscopic growth pattern may be a strong candidate for predicting the prognosis of CRC.

As not all patients benefit from adjuvant chemotherapy, it is important to find a sensitive predictive factor for chemotherapeutic efficacy to guide treatment strategies. Thus, we further compared the OS, CSS and DFS among the three macroscopic growth patterns using Kaplan-Meier 


\section{Subgroup}

Male (expansive vs. ulcerative) Male (infiltrative vs. ulcerative) Female (expansive vs. ulcerative) Female (infiltrative vs. ulcerative) Age $<50$ years (expansive vs. ulcerative) Age $<50$ years (infiltrative vs. ulcerative) Age 51-65 years (expansive vs. ulcerative) Age 51-65 years (infiltrative vs. ulcerative) Age 66-75 years (expansive vs. ulcerative) Age 66-75 years (infiltrative vs. ulcerative) Age $>75$ years (expansive vs. ulcerative) Age $>75$ years (infiltrative vs. ulcerative) Well differentiated (expansive vs. ulcerative) Well differentiated (infiltrative vs. ulcerative) Moderate differentiated (expansive vs. ulcerative) Moderate differentiated (infiltrative vs. ulcerative) Poor differentiated (expansive vs. ulcerative) Poor differentiated (infiltrative vs. ulcerative) Nonmucinous (expansive vs. ulcerative) Nonmucinous (infiltrative vs. ulcerative) Mucinous (expansive vs. ulcerative) Mucinous (infiltrative vs. ulcerative) RCC (expansive vs. ulcerative) RCC (infiltrative vs. ulcerative) LCC (expansive vs. ulcerative) LCC (infiltrative vs. ulcerative) RECC (expansive vs. ulcerative) RECC (infiltrative vs. ulcerative) Venous positive (expansive vs. ulcerative) Venous positive (infiltrative vs. ulcerative) Venous negative (expansive vs. ulcerative) Venous negative (infiltrative vs. ulcerative) Perineural positive (expansive vs. ulcerative) Perineural positive (infiltrative vs. ulcerative) Perineural negative (expansive vs. ulcerative) Perineural negative (infiltrative vs. ulcerative) Tumor deposit present (expansive vs. ulcerative) Tumor deposit present (infiltrative vs. ulcerative) Tumor deposit absent (expansive vs. ulcerative) Tumor deposit absent (infiltrative vs. ulcerative)

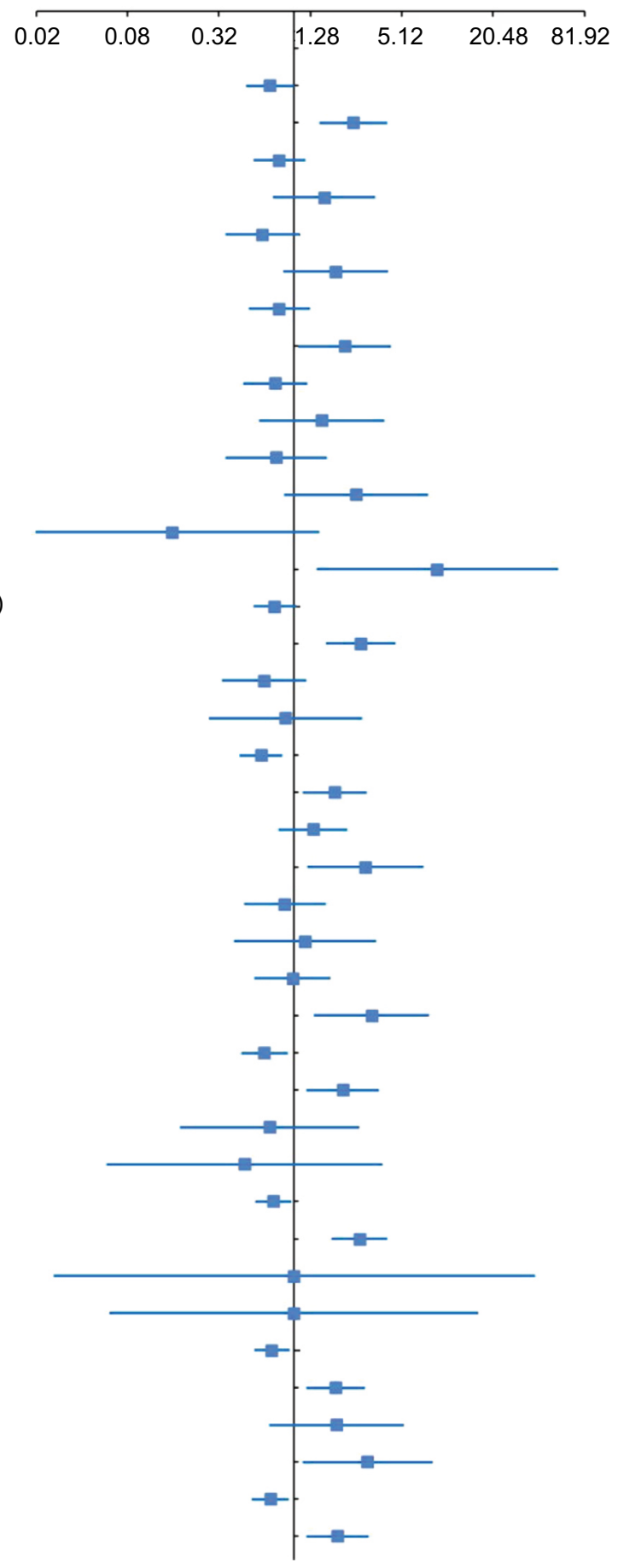

$\operatorname{HRs}(95 \% \mathrm{Cl})$

$0.696(0.481-1.007)$

$2.452(1.467-4.098)$

$0.801(0.541-1.187)$

$1.578(0.726-3.429)$

$0.619(0.352-1.089)$

$1.886(0.848-4.193)$

$0.798(0.501-1.269)$

$2.154(1.062-4.368)$

$0.752(0.461-1.226)$

$1.526(0.591-3.943)$

$0.762(0.354-1.639)$

$2.564(0.863-7.616)$

$0.158(0.017-1.478)$

$8.804(1.399-55.412)$

$0.745(0.537-1.034)$

2.744 (1.623-4.639)

$0.635(0.336-1.202)$

$0.881(0.275-2.818)$

$0.607(0.44-0.84)$

1.849 (1.135-3.01)

$1.332(0.795-2.233)$

2.938 (1.219-7.085)

0.867 (0.466-1.612)

$1.178(0.4-3.472)$

$0.983(0.551-1.753)$

$3.241(1.355-7.751)$

0.639 (0.449-0.908)

2.095 (1.208-3.635)

0.689 (0.176-2.693)

$0.474(0.058-3.858)$

$0.731(0.554-0.963)$

2.696 (1.773-4.1)

$1(0.026-39.006)$

1 (0.061-16.29)

$0.715(0.545-0.938)$

$1.882(1.21-2.926)$

$1.914(0.69-5.316)$

3.059 (1.134-8.249)

$0.699(0.528-0.924)$

$1.938(1.207-3.111)$

Figure 5 Adjusted HRs with $95 \%$ Cls for CSS comparing the infiltrative subtype and the expansive subtype to the ulcerative subtype in different subgroups. Abbreviations: CSS, cancer-specific survival; RCC, right colon cancer; LCC, left colon cancer; RECC, rectal cancer.

curves for patients with stage III disease. The results showed that patients with the expansive or ulcerative subtype had improved OS, CSS and DFS after the administration of chemotherapy, whereas patients with the infiltrative subtype had similar OS, CSS and DFS after the administration of chemotherapy. Thus, it is not recommended that patients with the infiltrative subtype receive chemotherapy because patients may not benefit from chemotherapy but rather suffer from it. However, the suitable treatment strategy for these patients needs to be further investigated.

To our knowledge, few studies have evaluated the prognostic and predictive value of the macroscopic growth pattern in CRC with different TNM stages and other subgroups. Our findings reveal the potential importance of evaluating 


\section{Subgroup}

Male (expansive vs. ulcerative) Male (infiltrative vs. ulcerative) Female (expansive vs. ulcerative) Female (infiltrative vs. ulcerative) Age $<50$ years (expansive vs. ulcerative) Age $<50$ years (infiltrative vs. ulcerative) Age 51-65 years (expansive vs. ulcerative) Age 51-65 years (infiltrative vs. ulcerative) Age 66-75 years (expansive vs. ulcerative) Age 66-75 years (infiltrative vs. ulcerative) Age $>75$ years (expansive vs. ulcerative) Age $>75$ years (infiltrative vs. ulcerative ) Well differentiated (expansive vs. ulcerative) Well differentiated (infiltrative vs. ulcerative) Moderate differentiated (expansive vs. ulcerative) Moderate differentiated (infiltrative vs. ulcerative) Poor differentiated (expansive vs. ulcerative) Poor differentiated (infiltrative vs. ulcerative) Nonmucinous (expansive vs. ulcerative) Nonmucinous (infiltrative vs. ulcerative) Mucinous (expansive vs. ulcerative) Mucinous (infiltrative vs. ulcerative) RCC (expansive vs. ulcerative) RCC (infiltrative vs. ulcerative) LCC (expansive vs. ulcerative) LCC (infiltrative vs. ulcerative) RECC (expansive vs. ulcerative) RECC (infiltrative vs. ulcerative) Venous positive (expansive vs. ulcerative) Venous positive (infiltrative vs. ulcerative) Venous negative (expansive vs. ulcerative) Venous negative (infiltrative vs. ulcerative) Perineural positive (expansive vs. ulcerative) Perineural positive (infiltrative vs. ulcerative) Perineural negative (expansive vs. ulcerative) Perineural negative (infiltrative vs. ulcerative) Tumor deposit present (expansive vs. ulcerative) Tumor deposit present (infiltrative vs. ulcerative) Tumor deposit absent (expansive vs. ulcerative) Tumor deposit absent (infiltrative vs. ulcerative)

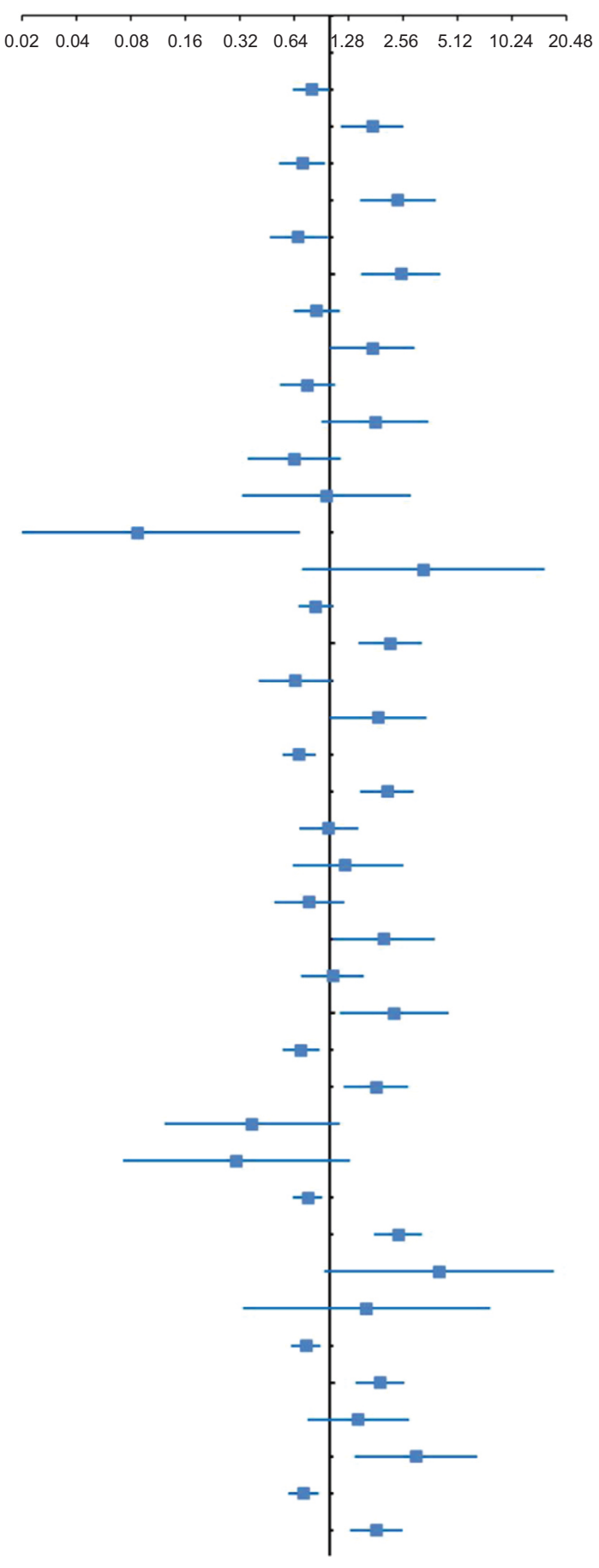

HRs $(95 \% \mathrm{Cl})$

$0.794(0.629-1.002)$

$1.724(1.154-2.576)$

$0.708(0.528-0.95)$

$2.395(1.481-3.874)$

$0.673(0.465-0.976)$

$2.488(1.503-4.118)$

$0.851(0.634-1.142)$

$1.724(1.004-2.959)$

$0.755(0.53-1.076)$

1.79 (0.906-3.536)

$0.638(0.352-1.155)$

$0.963(0.327-2.832)$

$0.087(0.011-0.687)$

$3.322(0.707-15.6)$

$0.838(0.676-1.038)$

$2.172(1.444-3.268)$

$0.646(0.408-1.024)$

$1.856(1.003-3.434)$

$0.681(0.551-0.842)$

$2.085(1.479-2.939)$

$0.992(0.683-1.441)$

$1.217(0.628-2.572)$

$0.774(0.497-1.206)$

$1.999(1.041-3.839)$

$1.042(0.699-1.554)$

$2.281(1.136-4.58)$

$0.695(0.55-0.877)$

$1.815(1.203-2.737)$

$0.373(0.122-1.141)$

$0.305(0.072-1.297)$

$0.76(0.631-0.915)$

$2.404(1.767-3.27)$

$4.045(0.94-17.415)$

$1.598(0.331-7.724)$

$0.74(0.615-0.889)$

1.905 (1.391-2.608)

$1.444(0.758-2.75)$

$3.013(1.38-6.576)$

$0.717(0.592-0.868)$

1.814 (1.294-2.545)

Figure 6 Adjusted HRs with $95 \%$ Cls for DFS comparing the infiltrative subtype and the expansive subtype to the ulcerative subtype in different subgroups. Abbreviations: DFS, disease-free survival; RCC, right colon cancer; LCC, left colon cancer; RECC, rectal cancer.

CRC prognosis and chemotherapeutic efficacy by combining macroscopic morphological features with other clinicopathological features in a relatively large Chinese cohort. However, there are several potential limitations of the present study that should be acknowledged. First, this study is a single-center study and additional multicenter studies are needed for further validation. Second, the present study is retrospective, and selection bias could not be completely avoided. Third, the patients recruited in this study are Chinese, and the application of the findings to other ethnic groups remains unclarified. Fourth, patients with tumors with an ambiguous appearance and stage IV CRC were excluded; therefore, we cannot give further recommendations for these patients. Further investigation should be conducted to examine whether 
the tumor growth pattern affects the survival and prognosis of these patients.

\section{Conclusion}

The macroscopic morphological subtype can be easily determined from medical records, and CRC with different growth patterns exhibits distinct clinical behaviors. The macroscopic growth pattern was an independent prognostic factor among stage I-III CRC patients in China. In addition, the infiltrative subtype had the worst DFS for stage II patients and the worst OS, CSS and DFS for stage III patients. Furthermore, the macroscopic growth pattern is able to predict the benefits of chemotherapy in stage III patients.

\section{Acknowledgments}

We wish to thank all participants for their assistance in this study, especially Zhenwei Shen and Jiqing Li for data collection and analysis. This work was supported by the National Natural Science Foundation of China (No. 81472685 and 81600469), the Major Special Plan of Science and Technology of Shandong Province (No. 2015ZDXX0802A01), the Science and Technology Development Projects of Shandong Province (No. 2016GSF201126) and the Natural Science Foundation of Shandong Province (No. ZR2016HB06 and ZR2017MH035).

\section{Author contributions}

All authors contributed toward data analysis, drafting and revising the paper and agree to be accountable for all aspects of the work.

\section{Disclosure}

The authors report no conflicts of interest in this work.

\section{References}

1. Torre LA, Bray F, Siegel RL, Ferlay J, Lortet-Tieulent J, Jemal A. Global cancer statistics, 2012. CA Cancer J Clin. 2015;65(2):87-108.

2. Chen W, Zheng R, Zeng H, Zhang S. The updated incidences and mortalities of major cancers in China, 2011. Chin J Cancer. 2015;34(11): 502-507.
3. Verdecchia A, Francisci S, Brenner H, et al; EUROCARE-4 Working Group. Recent cancer survival in Europe: a 2000-2002 period analysis of EUROCARE-4 data. Lancet Oncol. 2007;8(9):784-796.

4. Lin J, Qiu M, Xu R, Dobs AS. Comparison of survival and clinicopathologic features in colorectal cancer among African American, Caucasian, and Chinese patients treated in the United States: results from the Surveillance, Epidemiology and End Results (SEER) database. Oncotarget. 2015;6(32):33935-33943.

5. Edge SB, Compton CC. The American Joint Committee on Cancer: the 7th edition of the AJCC Cancer Staging Manual and the future of TNM. Ann Surg Oncol. 2010;17(6):1471-1474.

6. Jonker DJ, Spithoff K, Maroun J; Gastrointestinal Cancer Disease Site Group of Cancer Care Ontario's Program in Evidence-Based Care. Adjuvant systemic chemotherapy for stage II and III colon cancer after complete resection: an updated practice guideline. Clin Oncol (R Coll Radiol). 2011;23(5):314-322.

7. Liu F, Li C, Jia H, et al. Is there a prognostic value of tumor location among Chinese patients with colorectal cancer? Oncotarget. 2017;8(24): 38682-38692.

8. Zhou Y, Wang H, Gong H, Cao M, Zhang G, Wang Y. Clinical significance of perineural invasion in stages II and III colorectal cancer. Pathol Res Pract. 2015;211(11):839-844.

9. Wang JP. [Chinese standard for the diagnosis and treatment of colorectal cancer]. Zhonghua Wei Chang Wai Ke Za Zhi. 2011;14(1):1-4. Chinese.

10. Compton CC. Colorectal carcinoma: diagnostic, prognostic, and molecular features. Mod Pathol. 2003;16(4):376-388.

11. Vasile L, Olaru A, Munteanu M, Plesea IE, Surlin V, Tudoraşcu C. Prognosis of colorectal cancer: clinical, pathological and therapeutic correlation. Rom J Morphol Embryol. 2012;53(2):383-391.

12. Compton CC, Fielding LP, Burgart LJ, et al. Prognostic factors in colorectal cancer. College of American Pathologists Consensus Statement 1999. Arch Pathol Lab Med. 2000;124(7):979-994.

13. Kirzin S, Marisa L, Guimbaud R, et al. Sporadic early-onset colorectal cancer is a specific sub-type of cancer: a morphological, molecular and genetics study. PLoS One. 2014;9(8):e103159.

14. Dai W, Li Y, Meng X, Cai S, Li Q, Cai G. Does tumor size have its prognostic role in colorectal cancer? Re-evaluating its value in colorectal adenocarcinoma with different macroscopic growth pattern. Int J Surg. 2017;45:105-112.

15. Yang SH, Huang CJ, Chang SC, Lin JK. Association of C-reactive protein gene polymorphisms and colorectal cancer. Ann Surg Oncol. 2011;18(7):1907-1915.

16. Chen H, Wang Y, Liu H, et al. Factors influencing apical node metastasis in colorectal cancer patients treated with laparoscopic radical resection with D3 lymphadenectomy: results from two centers in China. Surg Today. 2015;45(5):569-575.

17. Chiang JM, Tan R, Wang JY, et al. S100P, a calcium-binding protein, is preferentially associated with the growth of polypoid tumors in colorectal cancer. Int J Mol Med. 2015;35(3):675-683.

18. Bosman FT, Carneiro F, Hruban RH, Theise ND. WHO Classification of Tumours of the Digestive System. Lyon: International Agency for Research on Cancer; 2010.
Cancer Management and Research

\section{Publish your work in this journal}

Cancer Management and Research is an international, peer-reviewed open access journal focusing on cancer research and the optimal use of preventative and integrated treatment interventions to achieve improved outcomes, enhanced survival and quality of life for the cancer patient. The manuscript management system is completely online and includes

\section{Dovepress}

a very quick and fair peer-review system, which is all easy to use. Visit $\mathrm{http}: / / \mathrm{www}$.dovepress.com/testimonials.php to read real quotes from published authors. 